\title{
Coordination of Rhythmic Motor Activity by Gradients of Synaptic Strength in a Neural Circuit That Couples Modular Neural Oscillators
}

\author{
Carmen Smarandache, ${ }^{1,2}$ Wendy M. Hall, ${ }^{1,2}$ and Brian Mulloney ${ }^{1,2}$ \\ ${ }^{1}$ Department of Neurobiology, Physiology, and Behavior, and ${ }^{2}$ Center for Neuroscience, University of California, Davis, Davis, California 95616-8519
}

\begin{abstract}
Synchronization of distributed neural circuits is required for many behavioral tasks, but the mechanisms that coordinate these circuits are largely unknown. The modular local circuits that control crayfish swimmerets are distributed in four segments of the CNS, but when the swimmeret system is active their outputs are synchronized with a stable intersegmental phase difference of 0.25 , an example of metachronal synchronization (Izhikevich, 2007). In each module, coordinating neurons encode detailed information about each cycle of the module's motor output as bursts of spikes, and their axons conduct this information to targets in other segments. This information is both necessary and sufficient for normal intersegmental coordination. In a comprehensive set of recordings, we mapped the synaptic connections of two types of coordinating neurons onto their common target neurons in other segments. Both types of coordinating axons caused large, brief EPSPs in their targets. The shape indices of these EPSPs are tuned to transmit the information from each axon precisely. In each target neuron's own module, these bursts of EPSPs modified the phase of the module's motor output. Each axon made its strongest synapse onto the target neuron in the nearest neighboring segment. Its synapses onto homologous targets in more remote segments were progressively weaker. Each target neuron decodes information from several coordinating axons, and the strengths of their synapses differ systematically. These differences in synaptic strength weight information from each segment differently, which might account for features of the system's characteristic metachronal synchronization.
\end{abstract}

\section{Introduction}

From a behavioral perspective, perhaps the most significant systems of segmental oscillators are those that drive locomotion (Grillner, 2006). Effective locomotion demands that movements of limbs be synchronized so that they move with the same period but at specific phases that define natural gaits. Moreover, limb movements must maintain these same phases despite changes in period that accompany changes in the animal's speed. In both arthropods (Wilson, 1961; Ikeda and Wiersma, 1964) and vertebrates (Grillner, 1981; Pearson, 1993; Skinner and Mulloney, 1998a), neural circuits within the CNS accomplish this synchronization by controlling the timing of bursts of action potentials (spikes) in motor neurons that innervate different muscles in each limb. These coordinating circuits make critical contributions to overt behaviors and have been studied at the systems level in many different animals (Orlovsky et al., 1999). However, in most cases their neural composition, synaptic organization, and dynamics are still poorly understood.

Received April 9, 2009; revised June 15, 2009; accepted June 22, 2009.

This work was supported by National Institutes of Health National Institute of Neurological Disorders and Stroke Grant NS048149. We thank Mark Goldman, Ann Hedrick, Tim Lewis, and Andy Ishida for reading the manuscript critically.

Correspondence should be addressed to Carmen Smarandache, Department of Neurobiology, Physiology, and Behavior, 196 Briggs Hall, University of California, Davis, One Shields Drive, Davis, CA 95616-8519. E-mail: csmarandache@ucdavis.edu.

DOI:10.1523/JNEUROSCI.1744-09.2009

Copyright $\odot 2009$ Society for Neuroscience $\quad$ 0270-6474/09/299351-10\$15.00/0
We investigated the synaptic organization of the neural circuit coordinating four pairs of modular local circuits that drive periodic movements of swimmerets in crayfish (Fig. 1). In vitro preparations of the crayfish ventral nerve cord will express coordinated bursts of spikes in different pools of swimmeret motor neurons (Ikeda and Wiersma, 1964) (Fig. 1C,D). The periods, the local phase differences between power-stroke (PS) and returnstroke (RS) bursts, and the posterior-to-anterior progression of PS bursts of this "fictive swimming" (Fig. $1 C$ ) are the same as the system's activity when the intact animal swims forward (Davis, 1968; Mulloney, 1997). Thus, the components of this coordinating circuit are present and functional and can be studied in these isolated nerve-cord preparations.

Each module includes a pattern-generating kernel of local neurons that organize firing of pools of swimmeret motor neurons (Figs. 1, 2). From each module, two neurons named $\mathrm{ASC}_{\mathrm{E}}$ and DSC project axons that distribute essential coordinating information to other modules (Stein, 1971; Namba and Mulloney, 1999; Tschuluun et al., 2001). In those target modules, these coordinating axons synapse onto local commissural neurons (one per target module) named ComInt 1 (Fig. 2C). ComInt 1 neurons integrate and transform this information, and transmit it to neurons in the local pattern-generating circuit within their own module (Mulloney and Hall, 2003).

We found that brief perturbations of a ComInt 1 neuron had a marked, phase-dependent effect on the timing of the motor output from its target module. Furthermore, spikes in both $\mathrm{ASC}_{\mathrm{E}}$ and DSC axons caused large, brief EPSPs in each ComInt 1 neu- 
ron. These EPSPs showed negligible short-term plasticity. In a comprehensive series of recordings from each ComInt 1 neuron, we observed systematic differences in strengths of EPSPs caused by coordinating axons from different modules. Each presynaptic axon encodes detailed information about each cycle of motor output from its home module. We conclude that the properties of their synapses onto ComInt 1 neurons are tuned to transmit that information precisely. However, each ComInt 1 neuron weights this information differently depending on the proximity of the module in which the presynaptic axon originates.

\section{Materials and Methods}

We anesthetized crayfish, Pacifastacus leniusculus, by chilling on ice and then exsanguinated them by transfusion with cold saline. To expose the abdominal CNS for recording, we removed the last two thoracic ganglia and the entire abdominal nerve cord (Fig. $1 B$ ) to a dish lined with transparent Sylgard (Dow-Corning) and pinned it out linearly dorsal side up with stainless steel pins. To permit insertion of microelectrodes, we used fine scissors to remove the sheath from the dorsal side of each ganglion.

Normal saline contained (in $\mathrm{mm}$ ) $5.4 \mathrm{KCl}$, 2.6 $\mathrm{MgCl}_{2}, 13.5 \mathrm{CaCl}_{2}$, and $195 \mathrm{NaCl}$, buffered with $10 \mathrm{~mm}$ Tris base and $4.7 \mathrm{~mm}$ maleic acid at $\mathrm{pH}$ 7.4. Low-Ca ${ }^{2+}$-high- $\mathrm{Mg}^{2+}$ saline contained (in mM) $5.4 \mathrm{KCl}, 52 \mathrm{MgCl}_{2}, 2.6 \mathrm{CaCl}_{2}$, and $118 \mathrm{NaCl}$, buffered with $10 \mathrm{~mm}$ Tris maleate at $\mathrm{pH}$ 7.4. If a preparation was not spontaneously active, we elicited stable expression of the swimmeret motor pattern by bath application of the cholinergic agonist carbachol (1.5-3 $\mu \mathrm{M}$ in different preparations) dissolved in normal saline (RBI, Sigma). The $\mathrm{ED}_{50}$ for carbachol's excitation of the swimmeret system is $7.8 \mu \mathrm{M}$ (Mulloney, 1997).

Electrophysiological recordings. Each pair of swimmerets is innervated by a pair of nerves (N1) that project bilaterally from the segmental ganglion directly to the swimmeret. Each N1 contains the motor and sensory axons that control one swimmeret. The axons of PS and RS motor neurons that project to each swimmeret are separated respectively into its N1's posterior and anterior branches (Mulloney and Hall, 2000). To record firing of PS and RS motor neurons from each ganglion, we placed extracellular stainless-steel pin electrodes in contact with these branches. We insulated each electrode from the bathing saline with a small amount of Vaseline.

The pairs of coordinating interneurons, $\mathrm{ASC}_{\mathrm{E}}$ and DSC, that originate in each module in ganglia $\mathrm{A} 2$ through $\mathrm{A} 5$ project their axons from the ganglion's lateral neuropils dorsally through the minuscule tract (MnT) toward the midline before entering the interganglionic connectives (Namba and Mulloney, 1999; Mulloney et al., 2003). We recorded action potentials in these axons extracellularly with a suction electrode placed on the $\mathrm{MnT}$ as it crossed dorsal to the lateral giant axon (Mulloney et al., 2003). Because of the details of the anatomy of these coordinating neurons and the swimmeret system (Namba and Mulloney, 1999; Mulloney et al., 2003), spikes recorded with suction electrodes positioned carefully on a left or right MnT could confidently be attributed to individual coordinating axons originating in the module just below that MnT.

Extracellular recordings were amplified and filtered using A-M Systems Model 1700 amplifiers. Microelectrode recordings were made using an npi SEC 05 amplifier (npi electronic). Signals from both sources were digitized using a Molecular Devices Digidata 1322A digitizer and pClamp software (Molecular Devices), and recorded as computer files for later analysis.

Microelectrodes contained 1\% Dextran Texas Red (Dextran Texas Red MW 3000 fixable; Invitrogen) dissolved in a solution of $1 \mathrm{M} \mathrm{K}$ acetate $+0.1 \mathrm{M}$ $\mathrm{KCl}$, and had resistance of 30-50 M $\Omega$. These electrodes were coated with Sylgard 186, except at the tip, to reduce capacitance. Individual ComInt 1 interneurons were penetrated at the midline in the minuscule tract (Skinner, 1985). Each neuron was first identified by physiological criteria given in Mulloney and Hall (2003). These identifications were confirmed independently by filling the neuron with Dextran Texas Red using $+1 \mathrm{nA}$ current pulses, $250 \mathrm{~ms}$ long at $2 \mathrm{~Hz}$. After the preparation was fixed overnight in $4 \%$ paraformaldehyde, the neuron's structure was examined in the cleared whole mount using a confocal microscope.

EPSPs were recorded using the npi amplifier's bridge mode. EPSCs were recorded using the npi amplifier's discontinuous single-electrode voltage-clamp mode (dSEVC). To permit high sampling rates, the microelectrodes were coated with Sylgard 186, and the level of the perfusion solution above the ganglion was kept low. The settling of the electrode voltage between each current pulse was monitored continuously on a dedicated oscilloscope. We usually attained switching frequencies above $20 \mathrm{kHz}$.

To explore the stability of transmission at synapses between coordinating axons and ComInt 1 neurons, we first found a ComInt 1 neuron and an individual coordinating axon from the neighboring ganglion in an active preparation. We then silenced the preparation by perfusing the preparation, except the ganglion containing the ComInt 1 neuron, with low-Ca ${ }^{2+}$-high- $\mathrm{Mg}^{2+}$ saline (Tschuluun et al., 2001). Once the preparation was quiet, pairs of spikes separated by controlled intervals were stimulated in the presynaptic axon by just-suprathreshold current pulses applied through the suction electrode. To test that any EPSPs recorded were caused by spikes in the previously identified presynaptic axons, we compared the delay between the stimulus and the EPSP with the delay 

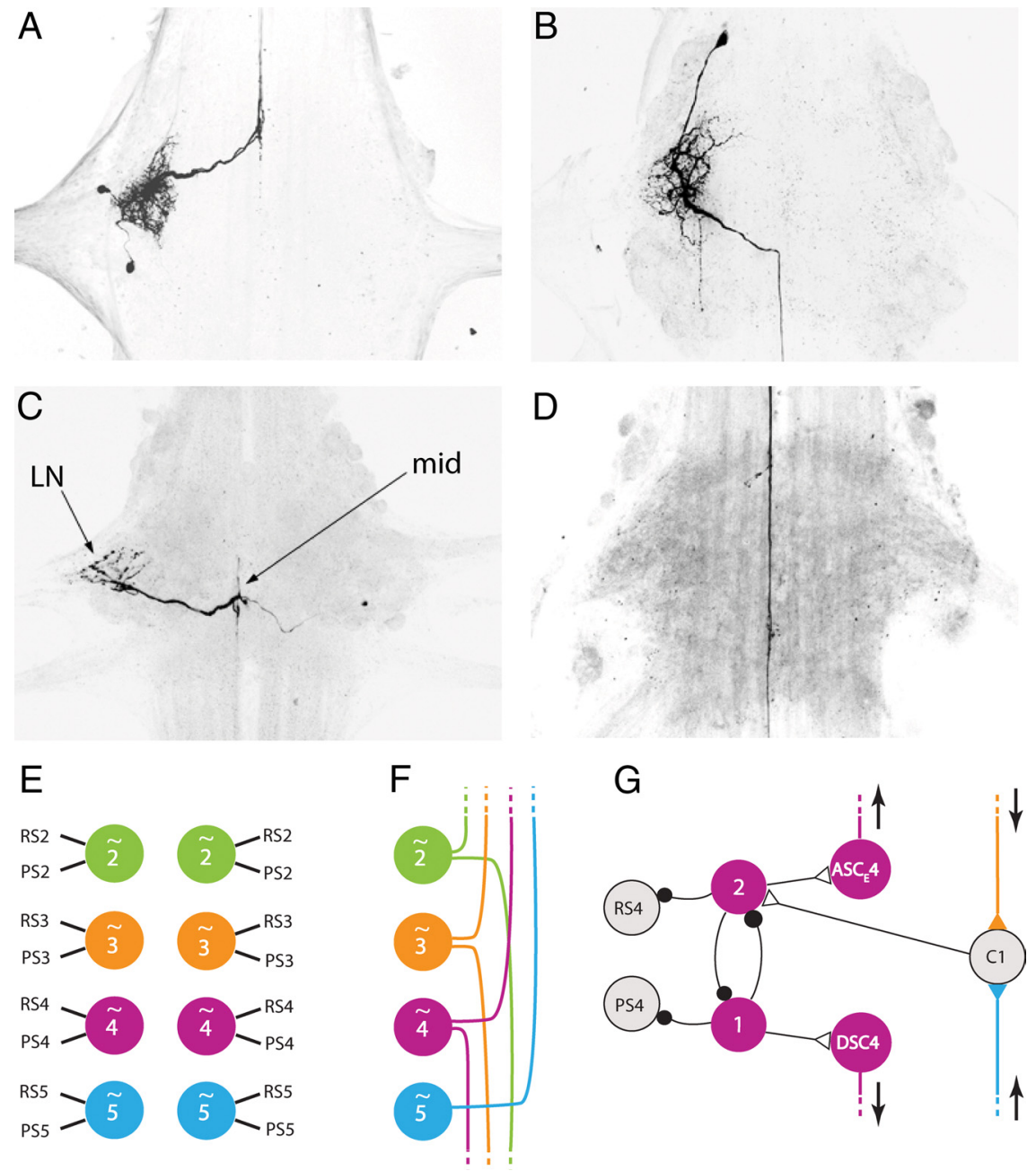

Figure 2. Neurons in the swimmeret coordinating circuit and diagrams of the system. These confocal stacks of whole mount preparations show entire ganglia, viewed from the dorsal side with anterior at the top. $A$, One $\operatorname{ASC}_{E}$ and one $\mathrm{ASC}_{L}$ neuron whose axons project from the lateral neuropil (LN) to exit the ganglion anteriorly. ${ }_{S} C_{L}$ neurons are not parts of the circuit discussed in this paper (Mulloney et al., 2006; Mulloney and Hall, 2007b). B, A DSC neuron whose axon projects posteriorly. C, A Comlnt 1 neuron with its cell body near the ganglion's right margin whose neurite crosses the midline (mid), extends a tuft of small branches, and continues to the opposite LN. D, A DSC axon filled in a ganglion posterior to its home ganglion. SC $_{E}$ axons course through the ganglia in the same tracts, and they have similar structures. $\boldsymbol{E}$, In each swimmeret segment $(2,3,4,5)$, a bilateral pair of local circuits $(\sim)$ control the firing of pools of PS and RS motor neurons that innervate each swimmeret. $\boldsymbol{F}$, From each local circuit, axons of two coordinating neurons project anteriorly ( $\mathrm{ASC}_{\mathrm{E}}$ ) or posteriorly (DSC) to the other ganglia. Here and in subsequent diagrams, only one side of the CNS or ganglion is illustrated. $G$, Within each local circuit, in this case $\sim 4$, the $\mathrm{ASC}_{\mathrm{E}}$ neuron and DSC neuron that originate there and project axons to other segments are driven by the same pattern-generating kernel that controls the swimmeret motor neurons (PS4, RS4). This kernel is composed of two sets of reciprocally inhibitor local interneurons (1, 2). Axons of ASC $_{E}$ and DSC neurons projecting from neighboring segments synapse onto commissural interneuron 1 (C1), which integrates the information they conduct and affects the oscillations of the module's kernel. In these diagrams, solid black circles symbolize inhibitory synapses, triangles symbolize excitatory synapses, the colors identify the segments in which axons originate, and arrows mark the direction of orthodromic impulse conduction.

between presynaptic spikes and EPSPs recorded when the system was active.

A facilitation index was calculated from paired-pulse experiments (Zucker and Regehr, 2002). The amplitudes of the first and second EPSPs in a series stimulated at the same interpulse interval were measured as the difference between the membrane potential before the EPSP and at its maximum (Stein et al., 2006). The mean amplitude and SD of each series were calculated using Excel (Microsoft Office Professional Edition 2003).

Shape indices of postsynaptic events. To measure the amplitudes, halfwidths, rise times, and decay times of synaptic potentials and currents, we triggered multiple recordings from ComInt 1 neurons using spontaneous spikes of a selected coordinated axon. Between 20 and 70 sweeps were averaged using Clampfit 10.2 (Molecular Devices). From this average waveform, Clampfit calculated the amplitude, half-width, and rise and decay times of the EPSP or EPSC. Rise time and decay time were measured from $10 \%$ to $90 \%$ of the peak. The means and SDs of these data were calculated using Excel (Microsoft).

Generating phase-response curves. Once an individual ComInt 1 had been identified, pulses of current were injected periodically to perturb the timing of the neuron's firing, using methods detailed in Mulloney and Hall (2007a). The durations of these pulses were 200 $\mathrm{ms}$, which approximates the durations of $\mathrm{ASC}_{\mathrm{E}}$ and PS bursts (Mulloney et al., 2006). The start and stop times of bursts of spikes in PS recordings from the neuron's home module were measured using Dataview (http://www. st-andrews.ac.uk/ wjh/) and used to describe the temporal structure of the module's motor output. The period of each cycle was the interval from the start of one PS burst to the start of the next PS burst (Fig. 1C,D). The latency of a stimulus occurring in a cycle was measured as the time interval between the start of the stimulus and the start of the preceding PS burst that marked the start of the cycle. The phases of these events then were defined as the ratio of these latencies to that cycle's period. Therefore, phase of a stimulus could range from 0 to 1.0.

To describe changes in the cycle periods caused by each pulse of injected current, we measured the periods of the four cycles that immediately preceded the start of the pulse and the period of the cycle in which the pulse occurred. For current pulse $i$ in cycle $j$, the mean of these four preceding periods, $\bar{X}_{i j}$, made a good predictor of the expected period following the start of the stimulus. For each stimulus, we calculated the normalized period difference, $\operatorname{Dif}_{i j}$, between each experimental period, $X_{i j}$, and the mean period just preceding it, $\bar{X}_{i j}$, as $\operatorname{Dif}_{i j}=\left(X_{i j}-\bar{X}_{i j}\right) / \bar{X}_{i j}$. Note that this definition of Dif $_{i j}$ is common among biologists and consistent with our prior work but opposite in sign to that common in mathematics (Canavier, 2006; Izhikevich, 2007). Plotting these $\mathrm{Dif}_{i j}$ as functions of the phase of the stimulus gives the phase6-response curve (PRC). We fitted a smoothed curve to each PRC using the "robust loess" function in MATLAB (MathWorks), taking into account that the $x$ values were not regularly spaced.

\section{Results}

Each cycle of swimmeret movements begins with a power stroke by the most posterior pair of swimmerets, and more anterior pairs follow with increasing phase delays. This same posterior-to-anterior progression is apparent in the motor output from isolated preparations of the ventral nerve cord (Fig. 1C). The swimmeret system includes four pairs of neural modules, one for each swimmeret (Murchison et al., 1993), located in abdominal ganglia A2, A3, A4, and A5 (Figs. $1 B, 2 E$ ). Each module includes $\sim 70$ PS and RS motor neurons that innervate one swimmeret (Mulloney and Hall, 2000). The kernel of each module is a pattern-generating circuit of local interneurons that controls firing of these motor neurons. 
Each module also has two coordinating neurons, $\mathrm{ASC}_{\mathrm{E}}$ and DSC, that send axons to other modules (Fig. 2) (Stein, 1971; Namba and Mulloney, 1999; Tschuluun et al., 2001). These coordinating neurons encode information about the timing, duration, and intensity of the module's motor output (Mulloney et al., 2006). In each module, PS motor neurons and the $\mathrm{ASC}_{\mathrm{E}}$ neuron fire bursts of spikes simultaneously (Fig. 1D). From the start time, duration, and number of spikes in each $\mathrm{ASC}_{\mathrm{E}}$ burst, a remote postsynaptic decoder could learn the start time, duration, and strength of the simultaneous burst in the PS motor pool (Mulloney et al., 2006). From each module, the $\mathrm{ASC}_{\mathrm{E}}$ 's axon projects anteriorly along the midline of the nerve cord at least as far as the thoracic ganglia (Figs. 1B, 2A) (Tschuluun et al., 2001). The second coordinating neuron, DSC, fires bursts at the same time as RS motor neurons, and encodes similar information about each RS burst (Fig. 1D). From each module, the DSC's axon projects posteriorly along the midline as far as ganglion A6 (Figs. 1 B, 2B) (Tschuluun et al., 2001).

As they traverse each ganglion (Fig. $2 D)$, both $\mathrm{ASC}_{\mathrm{E}}$ and DSC axons make en passant synapses onto local commissural neurons named ComInt 1 (Fig. 2C). ComInt 1 neurons decode the coordinating information transmitted by $\mathrm{ASC}_{\mathrm{E}}$ and DSC axons (Mulloney and Hall, 2003). ComInt $1 \mathrm{~s}$ are nonspiking neurons that occur as a bilateral pair in each ganglion (Fig. 2C). Each ComInt 1 receives synaptic input from $\mathrm{ASC}_{\mathrm{E}}$ and DSC axons at the midline (Fig. $2 C, D$ ), integrates that information, and transmits it through output synapses onto the local module in the lateral neuropil (Fig. 2C) (Mulloney and Hall, 2003). The output of each ComInt 1 neuron is restricted to one module, and has opposite effects on that module's PS and RS motor neurons (Mulloney and Hall, 2003). These differential effects result from a direct synaptic connection from ComInt 1 onto local neurons in the kernel of the module (Smarandache and Mulloney, unpublished).

What is the influence of transmission from ComInt 1 on the phase of its module's output? To measure this influence, we perturbed ComInt 1 with brief current pulses at different points in the module's output cycle and measured changes in the period of the perturbed cycle $[n=8$ experiments (expts)]. The PRCs that quantify these changes in period (Canavier, 2006) showed that ComInt 1 had significant effects on the module's period, and that these effects depended on the phase of the stimulus (Fig. 3). The period of the cycle increased if the depolarizing stimulus came early in the cycle, when PS motor neurons were firing, but decreased if the stimulus came later (Fig. $3 A$ ). Notice that hyperpolarizing currents in ComInt 1 also affected the module's period (Fig. 3B), and that the sign of this PRC is opposite that from depolarizing pulses. We think these results are due to changes in graded transmitter release from ComInt 1 caused by both depo-
B

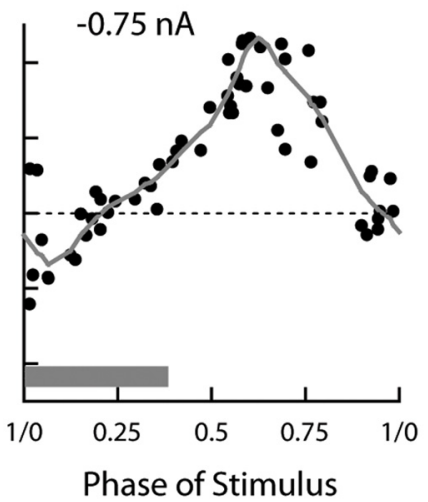

Phase of Stimulus

Phase of Stimulus

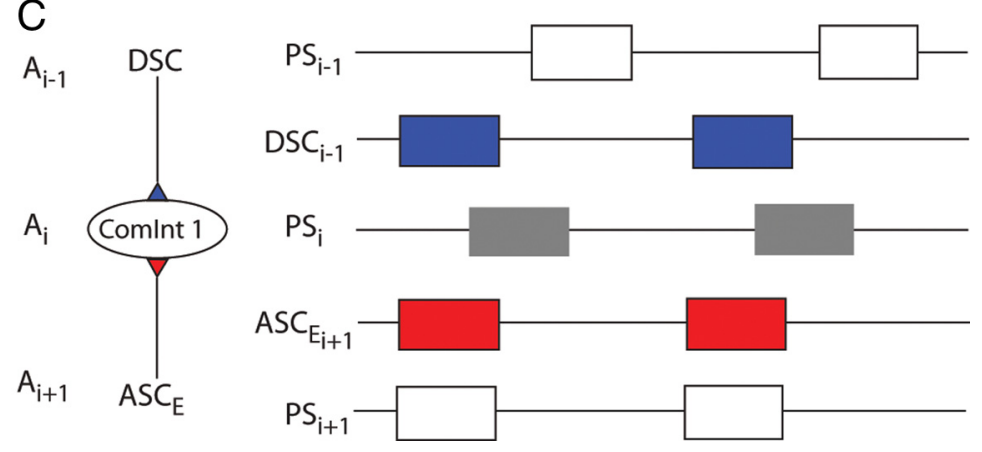

Figure 3. PRCs of PS bursts in response to current pulses delivered to the ComInt 1 neuron that projects to the same module (Fig. . These plots illustrate results of one experiment in which we used depolarizing current pulses $(\boldsymbol{A})$ and then hyperpolarizing pulses $(\boldsymbol{B})$ to perturb the same Comlnt 1 neuron. Normalized period difference is the difference between the measured period and 列 timing of ASC $_{E}$ and DSC bursts relative to PS bursts in their home modules (Fig. 1D), bursts of spikes in $A_{S C_{E i+1}}$ and DSC $C_{i-1}$ axons arrive simultaneously at the Comlnt 1 neuron in ganglion $A_{i}$.

larizing and hyperpolarizing current pulses. We note that the $\mathrm{PRCs}$ generated by stimulating individual $\mathrm{ASC}_{\mathrm{E}}$ or DSC axons are by comparison weaker and noisier than those generated by stimulating ComInt 1 directly (Mulloney and Hall, 2007a). From this evidence, we conclude that ComInt 1 neurons are key nodes through which the intersegmental phase of each ComInt 1's module's output can be tuned.

\section{Properties of synapses from coordinating axons onto ComInt 1 neurons}

Because of the $\sim 0.25$ phase differences between modules in neighboring segments and the different phases in each cycle at which $\mathrm{ASC}_{\mathrm{E}}$ and DSC neurons fire (Fig. $1 C, D$ ), bursts of spikes in $\mathrm{ASC}_{\mathrm{E}}$ and DSC axons from neighboring modules converge simultaneously on each ComInt 1 neuron (Fig. $3 C$ ). ASC $_{\mathrm{E}}$ axons encode variations in strengths of bursts of spikes in the PS motor neurons that control the force of power-stroke movements generating thrust for forward swimming. Under some conditions, this encoding is sufficiently precise that each additional $\mathrm{ASC}_{\mathrm{E}}$ spike in a burst correlates with a statistically significant increase in PS burst strength (Mulloney et al., 2006). We wanted to learn how the information encoded in these bursts is decoded. To begin, we recorded intracellularly from ComInt 1 neurons at the midline of each swimmeret ganglion, near the ComInt 1's syn- 
A

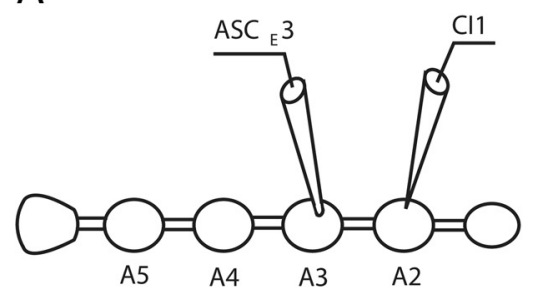

B

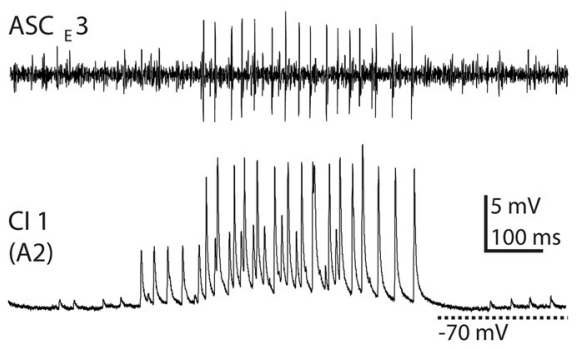

C
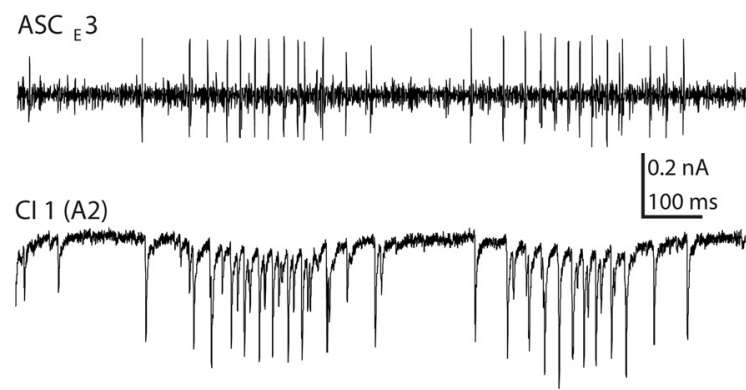

D

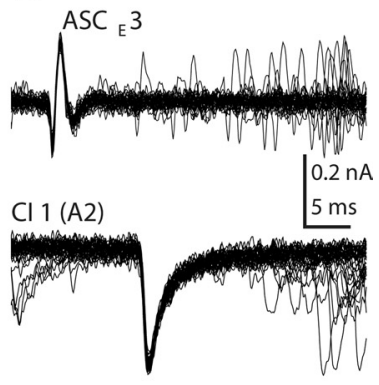

Figure 4. Recordings of postsynaptic potentials and currents in a Com Int 1 neuron in ganglion A2, the most anterior swimmeret ganglion. $A$, A diagram that shows the positions of recording electrodes. CI 1, An intracellular microelectrode in ComInt 1 at the midline of the ganglion, where this neuron receives synaptic input from coordinating axons (Fig. $2 C, D$ ). $\mathrm{ASC}_{\mathrm{E}}$, Extracellular recording from an $\mathrm{ASC}_{\mathrm{E}}$ axon originating in ganglion $\mathrm{A} 3$. $\boldsymbol{B}$, Simultaneous recordings of EPSPs in an $\mathrm{A} 2$ Comlnt 1 neuron and a burst of spikes in $\mathrm{ASC}_{\mathrm{E}} 3$ during expression of the swimmeret motor pattern (Fig. $\left.1 C, D\right)$. C, Simultaneous recordings of bursts of spikes in $\mathrm{ASC}_{\mathrm{E}} 3$ and PSCs they cause in a second A2 Comlnt 1.D, Multiple recordings triggered from $\mathrm{ASC}_{\mathrm{E}} 3$ spikes show the time-locked PSCS in this Comlnt 1 neuron.

A

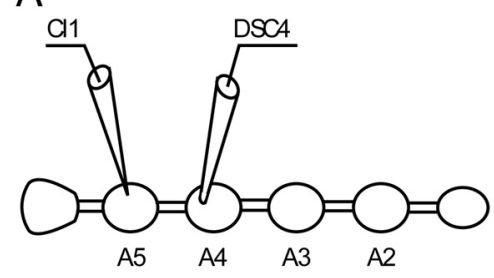

C
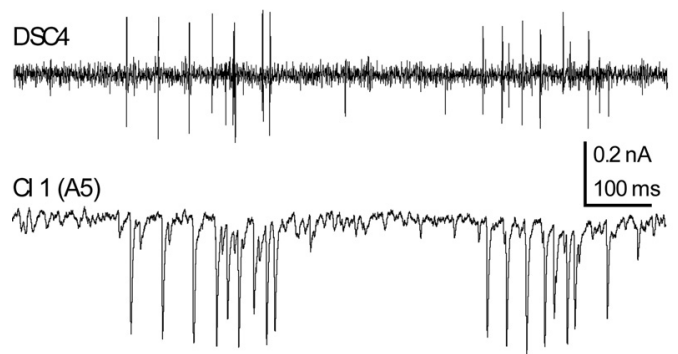
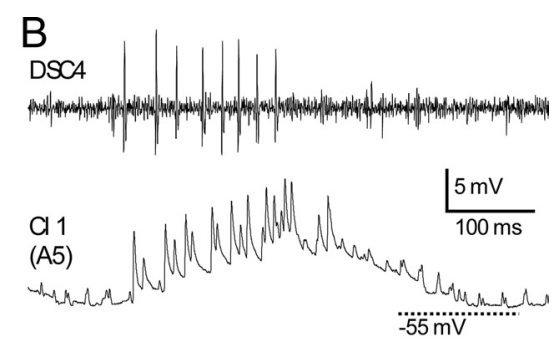

$D \mathrm{DSC}$

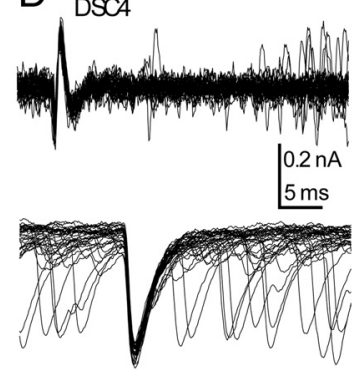

Figure 5. Postsynaptic potentials and currents recorded in a Comlnt 1 neuron in ganglion $A 5$, the most posterior swimmeret ganglion. $A$, A diagram showing positions of recording electrodes. Cl 1, An intracellular microelectrode in ComInt 1 at the midline of the ganglion, where this neuron receives synaptic input from coordinating axons from other ganglia. DSC4, Extracellular recording from the DSC axon originating in ganglion A4. B, Simultaneous recordings of EPSPs in an A5 Comlnt 1 neuron and a burst of spikes in DSC4. C, Simultaneous recordings of bursts of spikes in DSC4 and the PSCs these spikes cause in an A5 ComInt 1 neuron. D, Multiple recordings triggered by DSC 4 spikes show the time-locked PSCs in this A5 Comlnt 1.

apses with coordinating axons (Fig. 2), and extracellularly from coordinating axons that originated in other ganglia (Figs. 4-6).

When the system was active, ComInt 1 neurons in each module received periodic bursts of EPSPs of several sizes. When we triggered recordings from ComInt 1 neurons in A2 on spikes of
$\mathrm{ASC}_{\mathrm{E}}$ axons (Fig. 4), we found that each $\mathrm{ASC}_{\mathrm{E}}$ axon caused discrete EPSPs. These EPSPs were all very brief, and the shape indices of EPSPs from axons originating in different segments were not significantly different (Table 1).

When we triggered recordings from ComInt 1 neurons in A5 on spikes in DSC axons (Fig. 6), we found that each DSC caused discrete EPSPs. Like EPSPs caused by $\mathrm{ASC}_{\mathrm{E}}$ axons in more anterior ComInt 1 neurons, these EPSPs were very fast (Table 1). Both $\mathrm{ASC}_{\mathrm{E}}$ and DSC EPSPs were blocked by perfusion with low- $\mathrm{Ca}^{2+}$ high- $\mathrm{Mg}^{2+}$ saline solution (data not shown, $n=7$ expts).

To examine the synaptic currents that caused these fast EPSPs, we used dSEVC and sharp microelectrodes inserted at the midline (see Materials and Methods). Currents caused by neighboring $\mathrm{ASC}_{\mathrm{E}}$ spikes (Fig. $4 C, D$ ) had rise times of $0.7 \pm$ $0.1 \mathrm{~ms}$, half-widths of $2.4 \pm 0.9 \mathrm{~ms}$, and decay times of $8.4 \pm 4.1 \mathrm{~ms}$ (mean $\pm \mathrm{SD}$, $n=3$ expts). Currents caused by neighboring DSC spikes (Fig. $5 C, D$ ) had rise times of $0.7 \pm 0.1 \mathrm{~ms}$, half-widths of $2.3 \pm$ $0.9 \mathrm{~ms}$, and decay times of $8.6 \pm 5.4 \mathrm{~ms}$ ( $n=3$ expts). The similarities of these kinetic features of postsynaptic currents suggest that transmitter released from $\mathrm{ASC}_{\mathrm{E}}$ and DSC axons is detected by the same receptors.

\section{Amplitudes of EPSPs from different coordinating axons}

Because $\mathrm{ASC}_{\mathrm{E}}$ and DSC neurons originate in each module and project axons to all other swimmeret ganglia (Fig. 2F), ComInt 1 neurons in any one ganglion received a unique combination of EPSPs (Fig. 6, Table 2). ComInt 1 in A2 received only ascending coordinating information (Fig. 6B), while ComInt 1 in A5 received only descending coordinating information (Fig. $6 \mathrm{~K}$ ). In A3 and A4, ComInt 1 received a mixed input of ascending and descending information (Fig. 6E, H). By recording from ComInt 1 neurons in each ganglion, we could demonstrate that the largest synaptic input usually came from the $\mathrm{ASC}_{\mathrm{E}}$ that arose in the next posterior ganglion (Table 2).

In ganglion $\mathrm{A} 2$, ComInt 1 received input from $\mathrm{ASC}_{\mathrm{E}}$ axons originating in $\mathrm{A} 3$, A4, and A5 (Fig. 6B, C). The largest EPSPs in ComInt 1 neurons in A2 were caused by $\mathrm{ASC}_{\mathrm{E}}$ axons from $\mathrm{A} 3$, the neighboring posterior ganglion (Table 2). EPSPs caused by spikes in $\mathrm{ASC}_{\mathrm{E}} 4$ axons were $\sim 60 \%$ smaller, and those from ASC $_{\mathrm{E}} 5$ axons were $90 \%$ smaller than those from A3, or were not detectable $(n=4$ expts). In ComInt 1 neurons in A3, the largest EPSP was from $\mathrm{ASC}_{\mathrm{E}} 4$ (Fig. $6 E, F$ ). The EPSP from $\mathrm{ASC}_{\mathrm{E}} 5$ was $90 \%$ smaller, and 

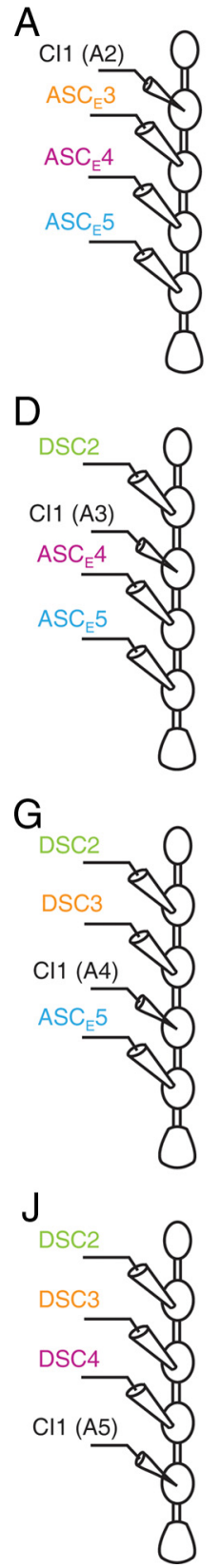

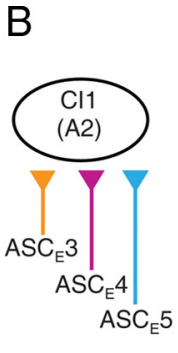

E

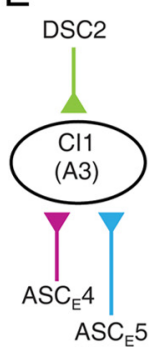

H

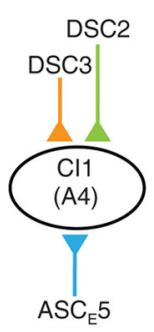

K

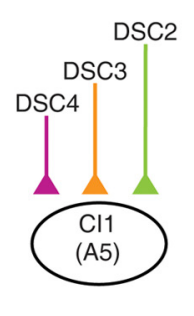

C

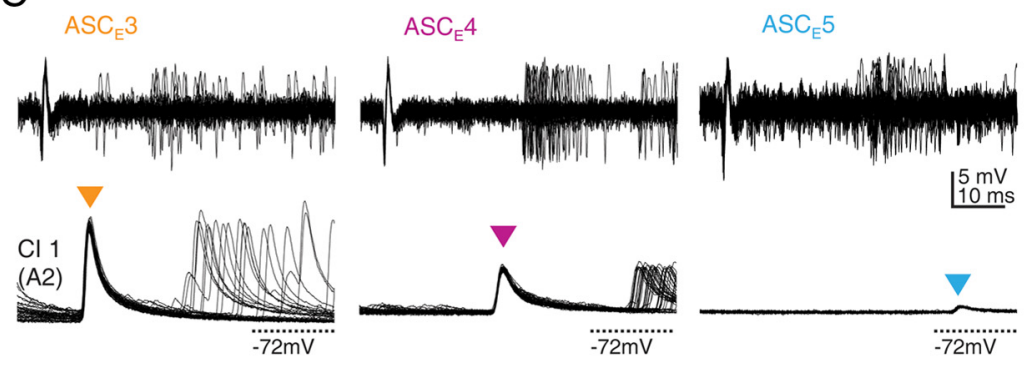

F
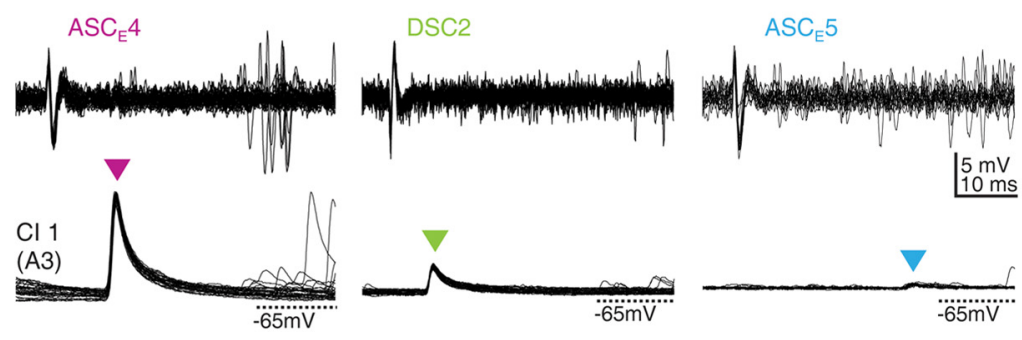

I
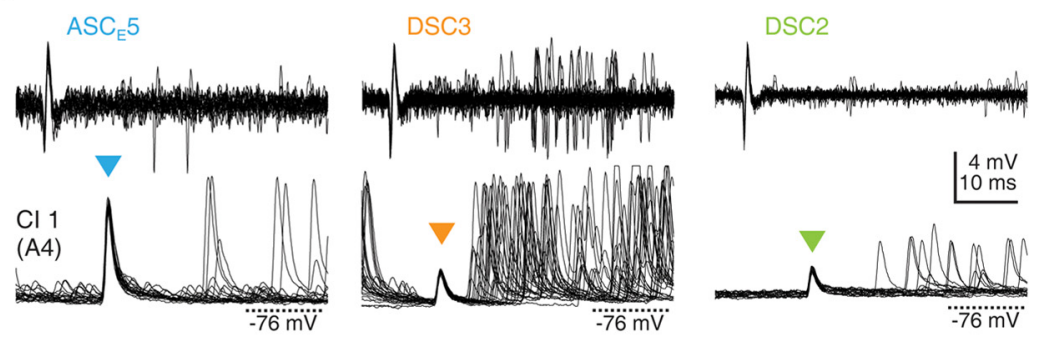

L
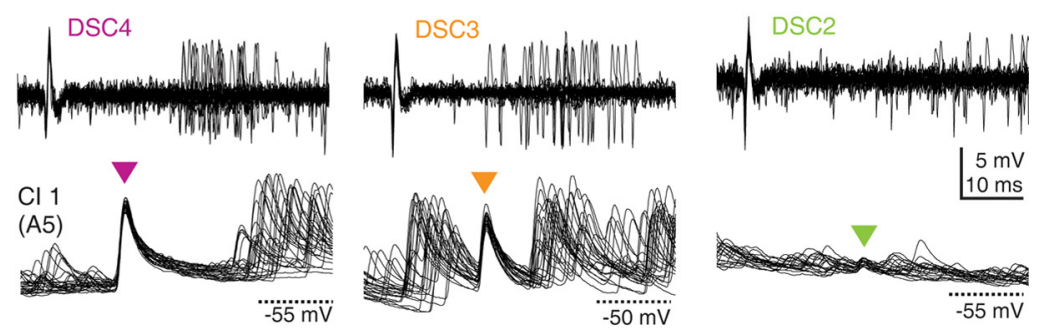

Figure 6. Segmental differences in the patterns and strengths of synaptic connections between coordinating axons and Comlnt 1 neurons. $A, D, G, J$, Diagrams that show positions of recording electrodes. Labels are the same as for Figures 4 and $5 . \boldsymbol{B}, \boldsymbol{E}, \boldsymbol{H}, \boldsymbol{K}$, Diagrams that summarize patterns of synaptic connections onto Comlnt 1 (CI1) neurons in ganglia A2, A3, A4, and A5. Triangles symbolize excitatory synapses. C, Simultaneous recordings of spikes in $\mathrm{ASC}_{\mathrm{E}} 3, \mathrm{ASC}_{\mathrm{E}} 4$, and $\mathrm{ASC}_{\mathrm{E}} 5$ and their EPSPs in the A2 Comlnt 1 neuron. The strongest EPSPS came from $\mathrm{ASC}_{\mathrm{E}} 3$. Comlnt $1 \mathrm{neurons}$ in $\mathrm{A2}$ receive no input from DSC neurons (Mulloney et al., 2006).F, Simultaneous recordings spikes in DSC2, ASC 4 4, and ASC 5 and their EPSPs in the A3 Comlnt 1.I, Simultaneous recordings of spikes in DSC2, DSC3, and $\mathrm{ASC}_{\mathrm{F}} 5$ axons and their EPSPs in the A4 Comlnt 1 neuron. The strongest EPSPs come from ASC ${ }_{\mathrm{F}}$. L, Simultaneous recordings of spikes in DSC2, DSC3, and DSC 4 axons and their EPSPs in the A5 Comln 11 neuron. The strongest EPSPs came from DSC4. Comlnt 1 neurons in A5 receive no input from $A_{S} C_{E}$ axons that originate in other modules (Mulloney et al., 2006).

the EPSP from DSC2 $70 \%$ smaller than the largest. In ComInt 1 neurons in A4, the largest EPSPs were from $\mathrm{ASC}_{\mathrm{E}} 5$ (Fig. $6 \mathrm{G}$, $H)$. The mean EPSP from DSC3 was similar, although in 4 of 7 experiments the EPSP from DSC3 was smaller than that from $\mathrm{ASC}_{\mathrm{E}} 5$. The EPSP from DSC2 was $\sim 60 \%$ smaller. In ComInt 1 neurons in A5, the largest EPSPs were from DSC4 (Fig. $6 K, L$ ). EPSPs from DSC3 were $20 \%$ smaller, and those from DSC2 were $\sim 85 \%$ smaller than those from DSC4. In every ganglion, the EPSPs elicited by spikes in $\mathrm{ASC}_{\mathrm{E}}$ and DSC axons were as brief as those recorded in $\mathrm{A} 2$ and $\mathrm{A} 5$ (Figs. $4 \mathrm{~B}, 5 \mathrm{~B}, 6$ ), and their shape indices were not significantly different (data not shown).
In earlier experiments we searched for homologues of $\mathrm{ASC}_{\mathrm{E}}$ and DSC neurons in ganglia A1 and A6 using the same methods that worked well in ganglia A2 through A5, but saw no sign of them (Mulloney et al., 2006). If such neurons exist, they are silent when the swimmeret system is active, and do not contribute to the performance of the coordinating circuit. Thus, the array of connections illustrated in Figure 6 and Table 2 is a comprehensive catalog of the synapses made by these intersegmental axons in this coordinating circuit.

\section{Short-term plasticity of synapses onto ComInt 1 neurons}

To investigate the stability of transmission from coordinating axons to ComInt 1 neurons, we recorded EPSPs from ComInt 1 
Table 1. Shape indices of EPSPs from different presynaptic axons recorded in ComInt 1 neurons in ganglia A2 and A5

\begin{tabular}{|c|c|c|c|c|c|c|}
\hline & \multicolumn{3}{|c|}{ Comlnt 1 in A2 } & \multicolumn{3}{|c|}{ Comlnt 1 in A5 } \\
\hline & $\mathrm{ASC}_{\mathrm{E}} 3$ & $\mathrm{ASC}_{\mathrm{E}} 4$ & $\operatorname{ASC}_{\mathrm{E}} 5$ & DSC2 & DSC3 & DSC4 \\
\hline$n$ & 8 & 6 & 1 & 2 & 3 & 5 \\
\hline \multicolumn{7}{|l|}{ Half-width } \\
\hline $\begin{array}{l}\text { Mean } \pm \text { SD } \\
\text { Range }\end{array}$ & $3.3 \pm 0.9$ & $3.9 \pm 1.5$ & 3.8 & $\begin{array}{l}1.6 \pm 0.1 \\
(1.6-1.7)\end{array}$ & $\begin{array}{l}2.3 \pm 0.6 \\
(1.7-2.8)\end{array}$ & $4.7 \pm 2.8$ \\
\hline \multicolumn{7}{|l|}{ Rise time } \\
\hline $\begin{array}{l}\text { Mean } \pm \text { SD } \\
\text { Range }\end{array}$ & $0.9 \pm 0.2$ & $1.1 \pm 0.4$ & 1.0 & $\begin{array}{l}0.7 \pm 0.1 \\
(0.6-0.7)\end{array}$ & $\begin{array}{l}0.8 \pm 0.1 \\
(0.7-0.9)\end{array}$ & $1.0 \pm 0.3$ \\
\hline
\end{tabular}

Values are given in milliseconds. $n$, Number of experiments. For indices based on small samples ( $n=2$ or 3 ), the range of measured values is given in parentheses.

elicited by stimulating individual axons from nearestneighboring ganglia, which we expected to make the strongest synapses, using a paired-pulse protocol (Zucker and Regehr, 2002). These data allowed us to calculate a facilitation index, the ratio of the size of the second EPSP to the first EPSP, and to see how this index changed as interspike interval changed (Fig. 7). We tested $\mathrm{ASC}_{\mathrm{E}}$ axons ( $n=5$ expts) and DSC axons ( $n=3$ expts) through a range of intervals from 10 to $100 \mathrm{~ms}$. Transmission here was quite stable; we saw no evidence of facilitation, and only slight depression of the second EPSP in pairs separated by $<20$ ms (Fig. 7C). This stability persisted during trains of 10 stimuli at $50 \mathrm{~Hz}$, structured to match bursts of spikes in $\mathrm{ASC}_{\mathrm{E}}$ axons during spontaneous expression of the swimmeret motor pattern (Fig. 1D) (Mulloney et al., 2006). Thus, these EPSPs from coordinating axons are brief, and very stable.

\section{Discussion}

Many behavioral and cognitive functions require synchronization of local circuits in different regions of the brain (Ikegaya et al., 2004). For the most part, the cellular mechanisms that accomplish synchronization of distributed local circuits are unknown, and discovering these mechanisms is a fundamental challenge for neuroscience. Here, following Izhikevich (2007, Chapter 10), we say that two circuits are synchronous if they oscillate with the same period and a constant phase (Pikovsky and Rosenblum, 2007). Within a local circuit, electrical coupling through gap junctions can accomplish perfect synchrony of coupled neurons (Schneider et al., 2006; Mancilla et al., 2007). Reciprocal inhibition can establish anti-phase synchrony (phase $=0.5$ ) (e.g., Fig. $1 D$ ) or in-phase synchrony (phase $=0$ ) of distributed circuits, depending on the tuning of the intrinsic properties of the neurons and inhibitory synapses (Wang and Rinzel, 1992). Metachronous synchronization, in which circuits have the same period but stable phases substantially different from 0.0 or 0.5 (Winfree, 2001), is more problematic. Neural circuits that coordinate rhythmic segmental oscillators often display metachronal coordination whose behavioral significance is clear, and they are ideal models to use to discover mechanistic principles of synchronization (Grillner et al., 2005; Yuste et al., 2005; Grillner, 2006).

The swimmeret circuit transforms information about the state of each local module at two points (Fig. 8): first from a graded synaptic drive from nonspiking local neurons into a burst of spikes in a coordinating axon, then from a burst of postsynaptic currents (PSCs) into a change in ComInt 1's membrane potential (Figs. 4, 5) that modulates graded transmission to the kernel of each ComInt 1's target module. To explain how this circuit accomplishes metachronal coordination, we must learn how information is encoded in coordinating axons, decoded by
Table 2. Amplitudes of EPSPs in ComInt 1 neurons in ganglia A2 through A5 caused by $\mathrm{ASC}_{\mathrm{E}}$ or DSC axons that originated in other ganglia

\begin{tabular}{|c|c|c|c|c|c|c|}
\hline & $\mathrm{ASC}_{\mathrm{E}} 3$ & $\mathrm{ASC}_{\mathrm{E}} 4$ & $\mathrm{ASC}_{\mathrm{E}} 5$ & DSC2 & DSC3 & DSC4 \\
\hline \multicolumn{7}{|l|}{ Comlnt 1 in $\mathrm{A} 2$} \\
\hline Mean $\pm S D$ & $4.8 \pm 3.2$ & $2.5 \pm 2.4$ & 0.7 & - & - & - \\
\hline$n$ & 8 & 6 & 1 & & & \\
\hline \multicolumn{7}{|l|}{ Comlnt 1 in A3 } \\
\hline $\begin{array}{l}\text { Mean } \pm S D \\
n \\
\text { Range }\end{array}$ & - & $\begin{array}{l}10.2 \pm 5.2 \\
8\end{array}$ & $\begin{array}{l}1.2 \pm 1.1 \\
2 \\
(0.4-2.1)\end{array}$ & $\begin{array}{l}1.8 \pm 1.4 \\
5\end{array}$ & $\bullet$ & - \\
\hline \multicolumn{7}{|l|}{ Comlnt 1 in A4 } \\
\hline $\begin{array}{l}\text { Mean } \pm \text { SD } \\
n\end{array}$ & $\bullet$ & $\bullet$ & $\begin{array}{l}3.5 \pm 2.5 \\
8\end{array}$ & $\begin{array}{l}1.9 \pm 0.5 \\
4\end{array}$ & $\begin{array}{l}3.5 \pm 2.4 \\
8\end{array}$ & $\bullet$ \\
\hline \multicolumn{7}{|l|}{ Comlnt 1 in A5 } \\
\hline $\begin{array}{l}\text { Mean } \pm S D \\
n \\
\text { Range }\end{array}$ & $\bullet$ & $\bullet$ & $\bullet$ & $\begin{array}{l}0.8 \pm 0.4 \\
2 \\
(0.5-1.1)\end{array}$ & $\begin{array}{l}4.6 \pm 1.0 \\
3 \\
(3.8-5.8)\end{array}$ & $\begin{array}{l}5.5 \pm 1.2 \\
5\end{array}$ \\
\hline
\end{tabular}

Values are mean amplitudes $\pm S D$, in millivolts; $n$, number of experiments; $\bullet$, no connection. For amplitudes based on small samples ( $n=2$ or 3 ), the range of measured values is given in parentheses.
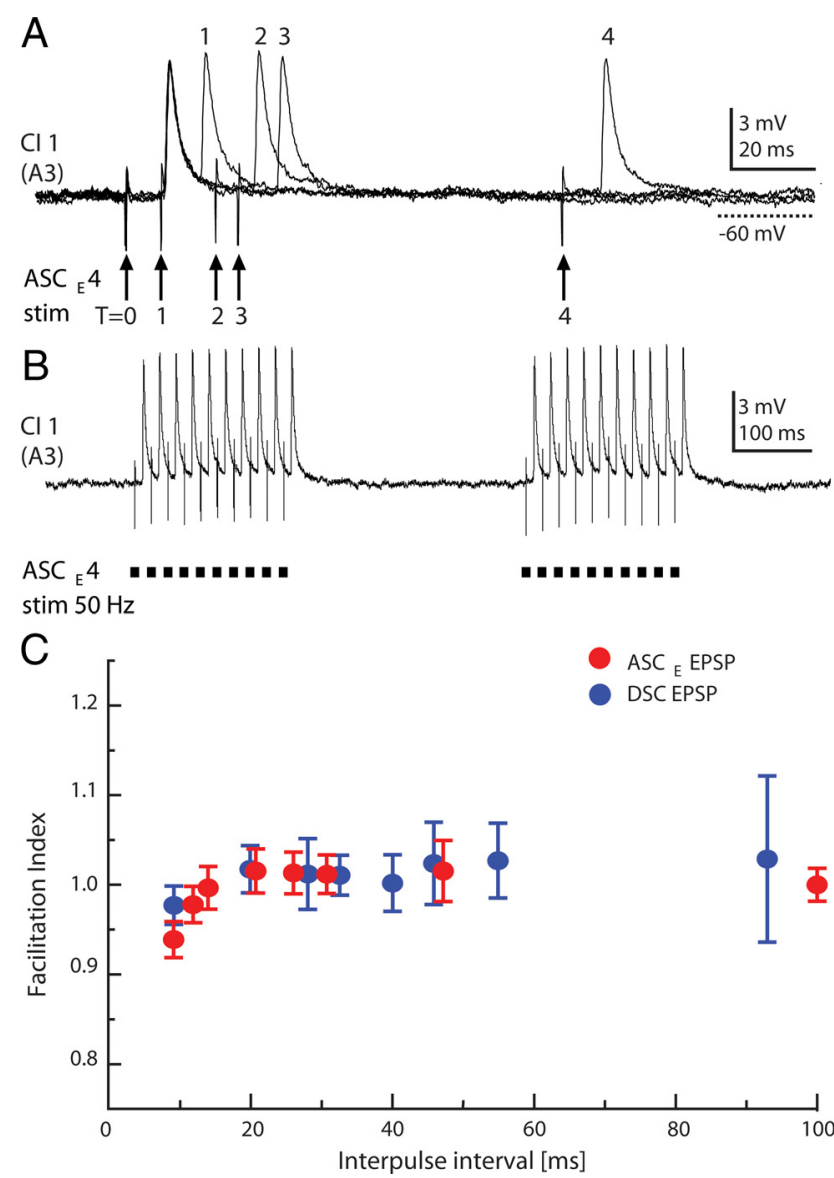

Figure 7. Absence of short-term plasticity in synapses between coordinating axons and ComInt 1 neurons. A, Paired-pulse stimulation of one ${ }^{A S C} C_{E}$ axon and simultaneous recordings of EPSPs in the Comlnt 1 neuron in its neighboring ganglion. In these four overlaid recordings, $T=0$ marks the first pulse of each pair. 1,2, 3, and 4 mark second pulses, delayed respectively by $8,20,25$, and $100 \mathrm{~ms}$, and the EPSPs they elicited. $B$, Two trains of stimuli at $50 \mathrm{~Hz}$. C, In this experiment, EPSPs elicited by stimulating identified $A_{S C} C_{E}$ and DSC axons were recorded in the same Com Int 1 neuron. The facilitation index (ratio of the second EPSP's amplitude to the first EPSP's amplitude) of pairs of $\mathrm{ASC}_{\mathrm{E}}$ and DSC EPSPs did not vary significantly with interpulse interval. Data points are means $\pm S D$. 


\section{Kernel of one module \\ Coordinating axon \\ Comlnt 1 neuron \\ Kernel of Comlnt 1's module}

Figure 8. A diagram illustrating the transformation of information from graded synaptic transmission within one local circuit into bursts of spikes in an intersegmental coordinating axon and then, through integration in a Comlnt 1 neuron, back into a graded transient depolarization that affects graded transmission to the kernel of the Comlnt 1 neuron's target module.

ComInt 1 neurons, and transmitted to local pattern-generating neurons.

The new description of the swimmeret coordinating circuit The results of these experiments (Fig. 6) enable us to construct the first comprehensive description of the cellular components and synaptic organization of the swimmeret coordinating circuit (Fig. 9). An $\mathrm{ASC}_{\mathrm{E}}$ neuron originates in each module in segments $\mathrm{A} 2$ through $\mathrm{A} 5$, and sends its axon anteriorly. The $\mathrm{ASC}_{\mathrm{E}}$ neuron fires simultaneously with PS motor neurons in its own module (Fig. 1D). A DSC neuron originates in each module in segments A2 through A4, and sends its axon posteriorly (Tschuluun et al., 2001; Mulloney et al., 2006). The DSC axon fires simultaneously with RS motor neurons in its own module (Fig. 1D). As they traverse each ganglion in the nerve cord, these axons synapse onto ComInt 1 neurons (Fig. 6). Spikes in these presynaptic axons cause EPSCs that ComInt 1 neurons integrate into transient depolarizations (Figs. 3C, 4, 5). Each ComInt 1 then transmits these periodic timing cues to its local neural oscillator (Fig. 3A).

When the system is active, modules in different ganglia have the same period because bursts of spikes in $\mathrm{ASC}_{\mathrm{E}}$ and DSC axons cause a burst of EPSPs in each ComInt 1 neuron. These periodic depolarizations of ComInt 1 neurons then entrain oscillations in the local pattern-generating circuits (Fig. 3) and so synchronize the chain of neural oscillators, as Coupled-Oscillator theory predicts (Kopell and Ermentrout, 1988; Skinner et al., 1997). PRCs that quantify the phase shifts of target modules caused by stimulating individual $\mathrm{ASC}_{\mathrm{E}}$ and DSC neurons show that both types of coordinating axons can do this (Mulloney and Hall, 2007a). Figures 2 and 3 are evidence that they do so through ComInt 1.

The integration of ascending and descending coupling information in one neuron to provide the timing cue to the local pattern-generating circuit and the convergence of information from more than nearest neighboring modules are features of this new description that were not anticipated in our previous models of this circuit (Skinner et al., 1997; Skinner and Mulloney, 1998b; Jones et al., 2003).

\section{Transmission and weighting of coordinating information}

Effective locomotion demands that the timing of movements on different segments of the body be coordinated. In vertebrates and arthropods, this coordination is accomplished by neural circuits intrinsic to the CNS (Marder and Bucher, 2001; Hill et al., 2003; Grillner, 2006; Kiehn, 2006). These circuits distribute information about each limb to other parts of the CNS: in vertebrates to other segments of the spinal cord and to the brainstem, in arthropods to other segmental ganglia. The bursts of spikes that occur in $\mathrm{ASC}_{\mathrm{E}}$ and DSC axons when the swimmeret system is active encode detailed information about each cycle of motor output from their home module (Mulloney et al., 2006). The properties of the synapses these axons make onto ComInt 1 neurons are well suited to transmit this detailed information with high fidelity (Figs. 4,
5). In principle, the brief EPSPs these spikes cause enable ComInt 1 neurons to count every spike in each presynaptic axon. If ComInt 1 neurons are simply low-pass filters whose output is some integral of their input, brief EPSPs are a puzzle. If, however, each EPSP affects transmission to ComInt 1's target more immediately, then individual EPSPs might be passed to the local pattern-generating circuit as discrete impulses.

The differences in strengths of synapses that each coordinating axon makes on the series of ComInt 1 neurons (Fig. 6, Table 2) weights the information it conducts differently in different
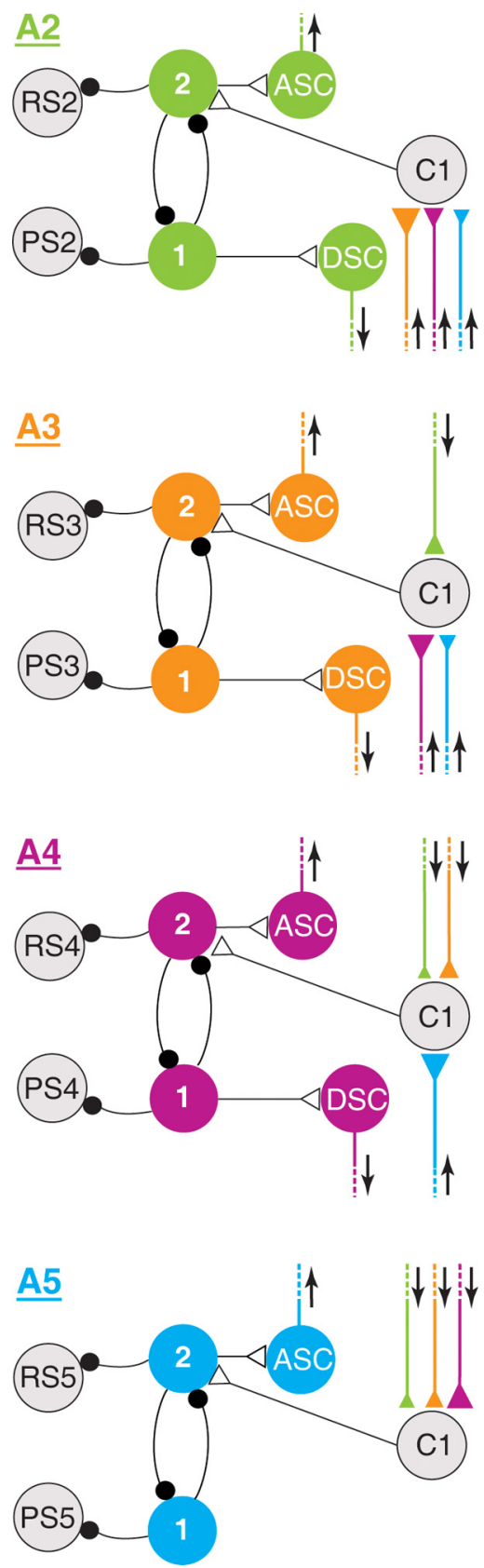

Figure 9. Diagrams of the synaptic organization of the intersegmental coordinating circuit in each swimmeret ganglion, $A 2, A 3, A 4$, and $A 5$. In each module, a ComInt 1 neuron (C1) integrates bursts of EPSPs from coordinating axons, transmits this information to neurons in the local pattern-generating circuit, and so adjusts the timing of the module's next PS burst. Symbols and colors are the same as in Figure 2. Different sizes of triangles indicate relative strengths of synapses. Arrows indicate the direction of impulse traffic in coordinating axons. 
modules. Each axon's influence will be greatest on the module in its neighboring ganglion. In more remote ganglia the same axon encoding the same information will have less influence. The noteworthy absence of short-term plasticity in these chemical synapses ensures that the relative strengths of synapses from different presynaptic axons remain distinct when changes in excitation of the system cause correlated changes in cycle period and presynaptic spike frequency (Mulloney et al., 2006). The highest spike-frequency observed in $\mathrm{ASC}_{\mathrm{E}}$ axons during expression of the normal swimmeret motor pattern was $\sim 65 \mathrm{~Hz}$ (Mulloney et al., 2006), and even at that frequency these synapses show no significant facilitation or depression (Fig. 7C).

The weaker synapses made by coordinating axons from more remote segments may explain an unusual failure of intersegmental coordination sometimes observed when synaptic transmission in ganglia A3 or A4 is blocked (Tschuluun et al., 2001). Modules on opposite sides of the block, A2 and A4 or A3 and A5 (Fig. $1 B$ ), were synchronized for many cycles, then synchronization would fail and they would oscillate at different periods with no preferred phase, but then synchronization would recover. Oscillators coupled by sufficiently weak connections would be operating at the limits of stability.

\section{Metachronal progression of the swimmeret motor pattern}

During forward swimming, each swimmeret makes alternating power strokes and return strokes that generate forward thrust. Swimmerets on different segments beat at the same frequency but with a characteristic phase delay from posterior to-anterior swimmerets. This phase difference is stable, and independent of frequency. These same features are apparent in the coordinated motor output recorded from swimmeret nerves in preparations of the isolated abdominal nerve cord (Fig. $1 B-D$ ). Ikeda and Wiersma (1964) showed that the most posterior ganglion does not contain a unique pacemaker for the system, so the posteriorto-anterior progression of PS bursts must emerge from properties of the coordinating circuit. The synaptic organization of this circuit (Fig. 9) and quantitative differences in synaptic strengths (Table 2) suggest an explanation for the posterior-to-anterior progression of each cycle of movements.

Gradients of excitability (Matsushima and Grillner, 1992) and gradients of excitation (Tunstall and Roberts, 1994) have been proposed to account for anterior-to-posterior phase progressions in spinal cord of swimming fish and tadpoles. There is no evidence of segmental gradients of excitability in the swimmeret system (Mulloney, 1997). There is, however, a segmental gradient in numbers of spikes per burst in coordinating neurons. Both $\mathrm{ASC}_{\mathrm{E}}$ and DSC neurons that originate in ganglia A5 and A4 fire more spikes per cycle than do those in A3 or A2 (Mulloney et al., 2006). These differences in numbers of spikes per burst might combine with differences in strengths of synapses to form a gradient of excitation. Since the strongest synapses in the circuit come from neighboring ganglia (Fig. 6, Table 2), ComInt 1 neurons in A4 and A5 will receive more EPSPs per cycle from their strongest synapses than do their counterparts in A3 or A2, a difference that could be considered a gradient of excitation reaching modules in different segments. We think this combination of more presynaptic spikes at stronger synapses in posterior modules might contribute to the normal posterior-to-anterior progression of swimmeret movements.

Segmental gradients of synaptic strength have been proposed to account for intersegmental phase differences in swimming fish (Williams, 1992; Tunstall and Roberts, 1994), and for features of the leech swim circuit (Friesen and Cang, 2001; Cang and Friesen,
2002) and leech heartbeat circuit (Masino and Calabrese, 2002; Hill et al., 2003). They have also been demonstrated in postsynaptic neurons that integrate information from crayfish stretchreceptor organs originating in different segments (Nakagawa and Mulloney, 2001). Here we have demonstrated the existence of such gradients in a multisegmental circuit by a comprehensive set of recordings from two kinds of coordinating axons and their target neurons in every segment of the swimmeret system (Fig. 6). This is the first demonstration of these phenomena in a neural circuit that coordinates limb movements.

\section{References}

Canavier CC (2006) Phase response curve. Scholarpedia 1:1332.

Cang J, Friesen WO (2002) Model for intersegmental coordination of leech swimming: central and sensory mechanisms. J Neurophysiol $87: 2760-2769$.

Davis WJ (1968) The neuromuscular basis of lobster swimmeret beating. J Exp Zool 168:363-378.

Friesen WO, Cang JH (2001) Sensory and central mechanisms control intersegmental coordination. Curr Opin Neurobiol 11:678-683.

Grillner S (1981) Control of locomotion in bipeds, tetrapods and fish. In: Handbook of physiology, the nervous system, Vol 2, Motor control (Brooks VB, ed), pp 1179-1236. Baltimore: Williams and Wilkens.

Grillner S (2006) Biological pattern generation: the cellular and computational logic of networks in motion. Neuron 52:751-766.

Grillner S, Kozlov A, Kotaleski JH (2005) Integrative neuroscience: linking levels of analyses. Curr Opin Neurobiol 15:614-621.

Hill AAV, Masino MA, Calabrese RL (2003) Intersegmental coordination of rhythmic motor patterns. J Neurophysiol 90:531-538.

Ikeda K, Wiersma CAG (1964) Autogenic rhythmicity in the abdominal ganglion of the crayfish: the control of swimmeret movements. Comp Biochem Physiol 12:107-115.

Ikegaya Y, Aaron G, Cossart R, Aronov D, Lampl I, Ferster D, Yuste R (2004) Synfire chains and cortical songs: temporal modules of cortical activity. Science 304:559-564.

Izhikevich EM (2007) Synchronization. In: Dynamical systems in neuroscience: the geometry of excitability and bursting, pp 443-505. Cambridge MA: MIT.

Jones SR, Mulloney B, Kaper TJ, Kopell N (2003) Coordination of cellular pattern-generating circuits that control limb movements: the sources of stable differences in intersegmental phase. J Neurosci 23:3457-3468.

Kiehn O (2006) Locomotor circuits in the mammalian spinal cord. Annu Rev Neurosci 29:279-306.

Kopell N, Ermentrout GB (1988) Coupled oscillators and the design of central pattern generators. Math Biosci 90:87-109.

Mancilla JG, Lewis TJ, Pinto DJ, Rinzel J, Connors BW (2007) Synchronization of electrically coupled pairs of inhibitory interneurons in neocortex. J Neurosci 27:2058-2073.

Marder E, Bucher D (2001) Central pattern generators and the control of rhythmic movements. Curr Biol 11:R986-R996.

Masino MA, Calabrese RL (2002) Phase relationships between segmentally organized oscillators in the leech heartbeat pattern generating network. J Neurophysiol 87:1572-1585.

Matsushima T, Grillner S (1992) Neural mechanisms of intersegmental coordination in lamprey: local excitability changes modify the phase coupling along the spinal cord. J Neurophysiol 67:373-388.

Mulloney B (1997) A test of the excitability-gradient hypothesis in the swimmeret system of crayfish. J Neurosci 17:1860-1868.

Mulloney B, Hall WM (2000) Functional organization of crayfish abdominal ganglia: III. Swimmeret motor neurons. J Comp Neurol 419:233-243.

Mulloney B, Hall WM (2003) Local commissural interneurons integrate information from intersegmental coordinating interneurons. J Comp Neurol 466:366-476.

Mulloney B, Hall WM (2007a) Local and intersegmental interactions of coordinating neurons and local circuits in the swimmeret system. J Neurophysiol 98:405-413.

Mulloney B, Hall WM (2007b) Not by spikes alone: responses of coordinating neurons and the swimmeret system to local differences in excitation. J Neurophysiol 97:436-450.

Mulloney B, Tschuluun N, Hall WM (2003) Architectonics of crayfish ganglia. Microsc Res Tech 60:253-265. 
Mulloney B, Harness PI, Hall WM (2006) Bursts of information: coordinating interneurons encode multiple parameters of a periodic motor pattern. J Neurophysiol 95:850-861.

Murchison D, Chrachri A, Mulloney B (1993) A separate local patterngenerating circuit controls the movements of each swimmeret in crayfish. J Neurophysiol 70:2620-2631.

Nakagawa H, Mulloney B (2001) A presynaptic basis for gradients in strength of synapses between different abdominal stretch-receptor axons and their common target neurons. J Neurosci 21:1645-1655.

Namba H, Mulloney B (1999) Coordination of limb movements: three types of intersegmental interneurons in the swimmeret system, and their responses to changes in excitation. J Neurophysiol 81:2437-2450.

Orlovsky GN, Deliagina TG, Grillner S (1999) Neuronal control of locomotion: from mollusc to man. Oxford: Oxford UP.

Pearson KG (1993) Common principles of motor control in vertebrates and invertebrates. Annu Rev Neurosci 16:265-297.

Pikovsky A, Rosenblum M (2007) Scholarpedia 2:1459.

Schneider AR, Lewis TJ, Rinzel J (2006) Effects of correlated input and electrical coupling on synchrony in fast-spiking cell networks. Neurocomputing 69:1125-1129.

Skinner FK, Mulloney B (1998a) Intersegmental coordination in invertebrates and vertebrates. Curr Op Neurobiol 8:725-732.

Skinner FK, Mulloney B (1998b) Intersegmental coordination of limb movements during locomotion: mathematical models predict circuits that drive swimmeret beating. J Neurosci 18:3831-3842.

Skinner FK, Kopell N, Mulloney B (1997) How does the crayfish swimmeret system work? Insights from nearest neighbor coupled oscillator models. J Comput Neurosci 4:151-160.

Skinner K (1985) The structure of the fourth abdominal ganglion of the crayfish, Procambarus clarkii. I. Tracts in the ganglionic core. J Comp Neurol 234:168-181

Stein PSG (1971) Intersegmental coordination of swimmeret motor neuron activity in crayfish. J Neurophysiol 34:310-318.

Stein W, Smarandache CR, Nickmann M, Hedrich UBS (2006) Functional consequences of activity-dependent synaptic enhancement at a crustacean neuromuscular junction. J Exp Biol 209:1285-1300.

Tschuluun N, Hall WM, Mulloney B (2001) Limb movements during locomotion: tests of a model of an intersegmental coordinating circuit. J Neurosci 21:7859-7869.

Tunstall MJ, Roberts A (1994) A longitudinal gradient of synaptic drive in the spinal cord of Xenopus embryos and its role in co-ordination of swimming. J Physiol 474:393-405.

Wang XJ, Rinzel J (1992) Alternating and synchronous rhythms in reciprocally inhibitory model neurons. Neural Comput 4:84-97.

Williams TL (1992) Phase coupling in simulated chains of coupled oscillators representing the lamprey spinal cord. Neural Comput 4:546-558.

Wilson DM (1961) The central nervous control of flight in a locust. J Exp Biol 38:471-490.

Winfree AT (2001) The geometry of biological time. Heidelberg: Springer.

Yuste R, MacLean JN, Smith J, Lansner A (2005) The cortex as a central pattern generator. Nat Rev Neurosci 6:477-483.

Zucker RS, Regehr WG (2002) Short-term synaptic plasticity. Annu Rev Physiol 64:355-405. 